\title{
Bioética, medicamentos, conflicto de intereses y control de calidad
}

\section{Bioethics, medicines, interests conflict and quality control}

\section{EMILIO LA ROSA RODRÍGUEZ*}

Resumen: El artículo analiza la problemática de la publicidad farmacéutica que deforma, exagera o esconde ciertas informaciones (efectos secundarios de los medicamentos), así como la labor de los visitadores médicos, el problema del riesgo medicamentoso, la autorización de comercialización, el acceso a los medicamentos, la practica terapéutica, los conflictos de intereses y el control de calidad en medicina. Estos temas tienen una importancia mayor en salud pública, ya que el no respetar los principios fundamentales de bioética (beneficencia, no maleficencia, autonomía y consentimiento) incide directamente sobre el estado de salud de la población.

Palabras clave: bioética - medicamentos - conflicto de intereses - control de calidad - riesgo - acceso - visitadores médicos - practica terapéutica

Summary: The article analyzes the problems of pharmaceutical advertising that distorts, exaggerates or hides certain information (side effects of drugs), as well as the work of pharmaceutical sales representatives, the problem of drugs' risk, marketing authorization, access to drugs, therapeutic practice, conflicts of interest, and quality control in medicine. These issues have a greater importance in public health; since the lack of respect for the fundamental principles of bioethics (benefit, not harm, autonomy and consent) has a direct impact on the health of the population.

Keywords: bioethics - drugs - conflict of interest - quality control - risk access - pharmaceutical sales representatives - therapeutic practice

CONTENIDO: I. LOS MEDICAMENTOS.- I.1. LA PUBLICIDAD FARMACÉUTICA.I.2. LOS VISITADORES MÉDICOS.- I.3. LOS MEDICAMENTOS Y EL RIESGO.I.4. LA AUTORIZACIÓN DE COMERCIALIZACIÓNY LAS NUEVAS MOLÉCULAS.I.5. EL ACCESO A LOS MEDICAMENTOS.- I.6. LA PRÁCTICA TERAPÉUTICA.II. LOS CONFLICTOS DE INTERESES.- III. EL CONTROL DE CALIDAD EN MEDICINA.- IV. CONCLUSIÓN.

* Director del «Centre de Recherche et d'Étude Santé et Société», París, Francia; vicepresidente del Comité Internacional de Bioética de la Unesco; médico; doctor en Antropología y Ecología Humana. Correo electrónico: elarosa@free.fr 


\section{LOS MEDICAMENTOS}

\section{I.1. La publicidad farmacéutica}

La publicidad farmacéutica viola ciertos principios éticos cuando transmite afirmaciones falsas sobre los fármacos, suprime u olvida informaciones sobre los riesgos y efectos secundarios, proporciona beneficios materiales (viajes, regalos, etcétera) o financieros a los médicos para que los prescriban, y utiliza la información sobre las enfermedades para promover los medicamentos y no para promover la salud $^{1}$. Es evidente que esta situación no es uniforme, sino que ella varía de país en país, con un denominador común: las mayores anomalías se dan en los países en desarrollo.

En 1985, la Organización Mundial de la Salud (OMS) estableció una serie de normas para la promoción ética de los medicamentos, pero estos criterios no tienen una obligación legal ${ }^{2}$. Según la OMS, la promoción de medicamentos debe ser precisa, veraz y demostrable, y no debe dar lugar a una utilización incorrecta o implicar riesgos innecesarios. La información sobre los medicamentos dirigida a los profesionales de la salud debe ser compatible con las pruebas y los resultados científicos demostrados y aprobados, y la información proporcionada al paciente debe ser redactada en términos comprensibles y reflejar adecuadamente los conocimientos médico-farmacológicos de acuerdo con las normas éticas $^{3}$. La legislación sanitaria debe tener en cuenta esta problemática, a fin de mejorar dicha información dentro del estricto respeto de los aspectos éticos de la promoción.

En ciertos países europeos ${ }^{4}$, la propia industria se autorregula porque los gobiernos no son muy activos para controlar los abusos. En algunos países de Asia, América Latina y África, este tipo de control es aún modesto, a causa de la corrupción y de los malos gobiernos. A pesar de que, tanto a nivel internacional y regional, como nacional, la industria farmacéutica ha establecido normas para hacer frente a estas actividades, en la práctica, las mismas no son aplicadas. 5 . Un estudio realizado en siete países europeos (República Checa, Dinamarca, Finlandia, Grecia, Hungría, Portugal y Eslovenia) pone de manifiesto

1 Ver Consumers International. «La promoción no ética de medicamentos» (http://www.consumidoresint. org/documentos/diamundial/WCRD_briefing_2007_spanish.pdf), 2007.

2 Ver Organisation Mondiale de la Santé. «Critères éthiques applicables à la promotion des médicaments, (http://mednet2.who.int/edmonitor/edition/edm17a.html), 1998.

3 Ibídem.

4 Ver Quick, Jonathan \& otros. «Ensuring Ethical Drug Promotion. Whose Responsibility?». Lancet, CCCLXII, 9385 (2003), p. 747

5 NoRRIS, Pauline \& otros. "Drug Promotion. What We Know, What We Have Yet to Learn. Reviews of Materials in the WHO/HAI Database on Drug Promotion» http://apps.who.int/medicinedocs/en/d Js8109e, 2004. 
las deficiencias del sector en la promoción de los medicamentos ${ }^{6}$. Los resultados de esta encuesta muestran lo siguiente:

- Una limitada transparencia en relación con los presupuestos dedicados a la publicidad. De todas las empresas farmacéuticas evaluadas, solamente una envió la información sobre su presupuesto publicitario.

- Nuevas estrategias publicitarias. Como la legislación europea no permite la publicidad dirigida al público de los medicamentos expedidos con receta médica, la industria farmacéutica orienta sus esfuerzos de promoción hacia las asociaciones de pacientes, los estudiantes de medicina y farmacia, los grupos de discusión por Internet, los sitios web sobre medicamentos, los folletos informativos sobre la salud y la enfermedad, y los artículos en revistas.

- Un incumplimiento de las normas sobre la promoción de los medicamentos. El estudio haidentificado una serie de infracciones graves, recientes y reiteradas, a las normas de la publicidad, especialmente en lo que respecta a medicamentos vendidos con receta médica. La actual normativa es insuficiente para prevenir violaciones sistemáticas de dichas normas.

Además, este mismo estudio señala que la industria farmacéutica no respeta sus propias normas de regulación sobre la promoción de los medicamentos, y que el sector farmacéutico no tiene como objetivo el interés de los consumidores, sino la generación de beneficios a través de la maximización de sus ventas. En este sentido, Consumers International indica que sin el control de las acciones poco éticas de promoción de los medicamentos, probablemente habrá un incremento del comportamiento irracional relativo a la prescripción, y un mal uso de los medicamentos por parte de los consumidores europeos?

\section{I.2. Los visitadores médicos}

En Francia, país en donde la reglamentación de la promoción de medicamentos es estricta, los visitadores médicos olvidan mencionar los efectos secundarios del medicamento, y en el $76 \%$ de los casos, es el médico el que hace preguntas sobre las contraindicaciones y las interacciones medicamentosas ${ }^{8}$. En un estudio realizado por

6 Ver Consumers International. La salud patentada. La perspectiva del consumidor sobre la RSE, la promoción de medicamentos y la industria farmacéutica en Europa. Londres: Consumers International, 2006, pp. 10-11 (http://www.consumersinternational.org/media/286105/salud_patentada.pdif).

7 Ver ibídem, p. 16.

8 HERMANGE, Marie-Thérèse \& Anne-Marie PAYET. «Rapport d'information fait au nom de la Commission des affaires sociales sur les conditions de mise sur le marché et de suivi des médicaments» (http:// www.senat.fr/rap/r05-382/r05-3820.html), 2006.

BIOÉTICA,

MEDICAMENTOS,

CONFLICTO DE

INTERESES Y

CONTROL DE

CALIDAD

BIOETHICS,

MEDICINES,

INTERESTS

CONFLICT AND

QUALITY CONTROL 
la Red del Observatorio de la Visita Médica de la revista Prescrire, se pudo constatar que solamente el 5\% de médicos recibió la información autorizada sobre un medicamento, y en el 35\% de los casos, la información proporcionada a los médicos miembros de la Red del Observatorio de la Visita Médica era diferente a aquella contenida en la Nota autorizada de medicamentos ${ }^{9}$. En realidad, se constató más bien una ampliación de las indicaciones y un aumento de la posología recomendada ${ }^{10}$. El aumento del volumen de venta de un medicamento puede llegar al 30\% cuando se hace esfuerzos de promoción a nivel del médico que prescribe ${ }^{11}$.

En la mayoría de los países, 80 a 95\% de los médicos reciben a los visitadores médicos sin tener en cuenta que la información que proporcionan es excesivamente favorable a los medicamentos ${ }^{12}$ y que generalmente no brindan información sobre los efectos secundarios de los fármacos. En resumen, la visita médica propone generalmente una información parcial e incompleta ${ }^{13}$. Además, existe una correlación significativa entre la frecuencia de las visitas y la prescripción irracional, por lo que creemos que este tipo de actividad no contribuye a una mejor atención médica, ya que su única razón de existir es la de vender medicamentos. La información parcializada facilitada a los médicos por la industria farmacéutica debe necesariamente ser equilibrada por otra independiente proporcionada por las autoridades sanitarias a través de mecanismos que permitan el fácil acceso a dicha información por parte del cuerpo médico.

Si la publicidad farmacéutica (incluyendo la visita médica) manipula, esconde o modifica la información sobre los medicamentos, ella está creando las condiciones necesarias para un uso no racional del medicamento. Sin embargo, la responsabilidad referente a la prescripción de medicamentos recae directamente sobre el médico.

\section{I.3. Los medicamentos y el riesgo}

En materia de riesgo, no se puede aceptar bajo ningún pretexto que un medicamento siga siendo comercializado si los resultados de los estudios demuestran que dicho medicamento tiene efectos nocivos graves para la salud. Es evidente que dicho fármaco deber ser inmediatamente retirado del mercado en cumplimiento con el principio de no maleficencia señalado en el artículo 4 de la Declaración

9 La Nota de medicamentos es un resumen de las características del producto y de las menciones legales. Este documento está destinado al médico.

10 Le Réseau d'observation de la visite médicale. "La visite médicale trompe toujours énormément». Prescrire, XXV, 259 (2005), p. 191.

11 Hermange, Marie-Thérèse \& Anne-Marie PAYET. Ob. cit.

12 LEXCHIN, Joel. «What Information do Physicians Receive from Pharmaceutical Representatives?». Canadian Family Physician, 43 (1997), pp. 941-945.

13 WAZANA, Ashley. «Physicians and the Pharmaceutical Industry: Is a Gift Ever Just a Gift?». Journal of the American Medical Association, CCLXXXIII, 3 (2000), pp. 373-380. 
Universal sobre Bioética y Derechos Humanos ${ }^{14}$, documento ratificado por el Gobierno peruano. Este principio es un concepto ancestral propuesto por Hipócrates en su famosa frase Primum non nocere («Ante todo, no hacer daño»), que preconiza el respeto a la integridad física y psicológica de la vida humana. Este concepto es de gran actualidad en razón del progreso científico y tecnológico que conlleva un cierto riesgo, y por la tendencia a proponer un medicamento (y el riesgo de los efectos secundarios inherentes a todo fármaco) como única alternativa o solución a ciertas situaciones, como son los síntomas banales, los síndromes mal definidos y nebulosos, la medicalización de la vida y los medicamentos de confort (medicamentos para el estilo de vida).

El principio de no maleficencia debe tener en cuenta el respeto del equilibrio daño-beneficio. Cuando este equilibrio se rompe, en razón de un beneficio mínimo o un riesgo elevado, es éticamente inaceptable hacer correr un riesgo inútil a la persona. Fatalmente, esta situación no es rara, los casos más conocidos fueron el del rofecoxib (Vioxx®) y el benflorex (Mediator $\left.{ }^{\circledR}\right)$. El Vioxx ${ }^{\circledR}$ fue comercializado en 1999 y retirado del mercado en septiembre de 2004 debido a los efectos nocivos graves que provocaba. Sin embargo, sus efectos graves se conocían desde el año 2002. Según un estudio realizado en los Estados Unidos de Norteamérica, el rofecoxib ha causado entre 88000 y 140000 casos de enfermedades coronarias. Los pacientes tratados con dicho fármaco presentaron un riesgo coronario multiplicado por 1,34 en relación con aquellos tratados con analgésicos convencionales. Este riesgo se presentaba a partir del tercer mes de tratamiento con el rofecoxib. A pesar de estas afirmaciones, hubo dificultades para publicar los resultados de esta investigación.

En Francia, el benflorex (Mediator®) es un anfetamínico comercializado en 1976 y retirado del mercado en noviembre de 2009. Los primeros indicios de efectos nocivos graves (lesiones graves de las válvulas cardiacas, hipertensión pulmonar) fueron publicados en 1997. Este mismo año, el benflorex fue retirado del mercado norteamericano, sin embargo, continuó su comercialización en otros países, especialmente en Francia. Esta larga comercialización ha provocado una catástrofe sanitaria: 500 a 1000 personas fallecidas a causa de los efectos nocivos graves de dicho medicamento.

La aplicación de los principios de precaución y de no maleficencia hubiesen sido más que suficiente para retirar el benflorex del mercado francés. Sin embargo, «en ningún momento durante este largo período, ningún médico experto de la agencia (de medicamentos) pudo conducir un razonamiento farmacológico adecuado que permita clarificar 
la decisión de las autoridades» ${ }^{15}$. Es decir que los médicos expertos no fueron capaces de realizar un trabajo eficaz y correcto, razón por la cual nos preguntamos si en este caso preciso hubo conflicto de intereses.

\section{I.4. La autorización de comercialización y las nuevas moléculas}

Los ensayos clínicos de un nuevo medicamento son requisitos indispensables para obtener la autorización de comercializarlo. Sin embargo, esos ensayos son realizados y financiados por el laboratorio que fabrica dicho medicamento. Muy a menudo, las autoridades sanitarias encargadas de dar dicha autorización no cuentan con ensayos clínicos independientes que le sirvan de referencia para evaluar la calidad y el beneficio terapéutico del nuevo medicamento. En otras palabras, la autorización está basada en la buena fe del laboratorio que puede esconder o manipular la información. Así en el caso del benfluorex, el laboratorio obvió el efecto anfetamínico de dicho medicamento, presentándolo como un medicamento de segunda línea para el tratamiento de la diabetes.

En el último decenio, la industria farmacéutica ha comercializado numerosos medicamentos que no aportan a un verdadero progreso terapéutico para los pacientes ${ }^{16}$. Sin embargo, los precios de esas nuevas moléculas son generalmente dos a tres veces más elevados que los de sus similares que se encuentran en el mercado. Nos preguntamos, icómo es posible poner en el mercado nuevos medicamentos que no representan un progreso terapéutico tangible para los pacientes a precios elevados?

Simplemente porque el principio de libre comercio se aplica también al sector farmacéutico y las autoridades sanitarias no pueden rechazar el pedido de comercialización de un nuevo medicamento, aun si este no posee un beneficio terapéutico superior a los ya existentes en el mercado. Si bien es cierto que no es posible rechazar esta autorización, sí es posible rechazar el precio de venta propuesto por el laboratorio farmacéutico. En otras palabras, si el nuevo medicamento no representa un progreso terapéutico superior a los ya existentes, su precio no debe multiplicarse por dos, tres o cuatro. Otra alternativa consiste en reevaluar la autorización de comercialización del medicamento al cabo de cinco años con la finalidad de hacer un análisis del beneficio-riesgo y del valor terapéutico agregado. Dicho valor terapéutico debe de ser publicado con la finalidad de mejorar la información, base fundamental

15 BENSADON, Anne-Carole \& otros. «Enquête sur le Mediator®. Rapport définitif». Inspection générale des affaires sociales RM2011-001P, 2011 (http://www.ladocumentationfrancaise.fr/var/storage/ rapports-publics/114000028/0000.pdf).

16 COLLECTIF EUROPE ET MÉDICAMENT. «Rendre publique la "valeur thérapeutique ajouté des nouveaux médicaments" »http://www.prescrire.org/docus/valeurAjouteeFr.pdi), 2003. 
del consentimiento libre preconizado por el artículo 6.1 de la Declaración Universal sobre Bioética y Derechos Humanos ${ }^{17}$.

\section{I.5. El acceso a los medicamentos}

El principio de justicia defiende la distribución y el acceso equitativo a los recursos sanitarios. El artículo 14.2 inciso «a» de la Declaración Universal reivindica este principio que propicia y defiende «el acceso a una atención médica de calidad y a los medicamentos esenciales, especialmente para la salud de las mujeres y los niños, ya que la salud es esencial para la vida misma y debe considerarse un bien social y humano». Sin embargo, estamos aún lejos de satisfacer las necesidades de la población en términos de acceso y de calidad de los medicamentos. Tenemos aún dificultades de acceso por razones geográficas y económicas.

El precio prohibitivo de ciertos medicamentos, sobre todo de los nuevos, es un obstáculo mayor, difícil de resolver en una economía de libre mercado. Sin embargo, el medicamento no es un bien exclusivamente comercial, sino también social y humano, porque participa en la recuperación de la salud y la prevención de la enfermedad individuales y colectivas. Esta dimensión social y humana es más que suficiente para que los insumos médicos, particularmente los medicamentos, sean tratados de forma diferente. En otras palabras, los precios de los medicamentos deben calcularse teniendo en cuenta no solamente el costo de investigación y desarrollo, sino también el beneficio terapéutico para el paciente. Muchas veces los costos de investigación y desarrollo de los nuevos medicamentos son exagerados, porque se incluye en dichos costos la fase 4 de los ensayos clínicos que sirve fundamentalmente para vender el medicamento.

La industria farmacéutica justifica los precios elevados de los medicamentos aduciendo que los costos de investigación y desarrollo son muy altos. En 1993, el Office of Tecnology Assessment (organismo creado por el Congreso de los Estados Unidos de Norteamérica, y que fue disuelto posteriormente) estimaba el costo promedio de un nuevo medicamento en 802 millones de dólares. Dicho costo incluye los estudios preclínicos, clínicos y los beneficios esperados si el laboratorio farmacéutico hubiera invertido ese capital en otro campo de investigación y desarrollo.

En el año 2006, Jürgen Drews ${ }^{18}$ consideraba que el costo promedio de un nuevo medicamento era de 1250 millones de dólares (359 millones de dólares en 1990 y 700 millones en 1997). En Francia,

17 «Toda intervención médica preventiva, diagnóstica y terapéutica solo habrá de llevarse a cabo previo consentimiento libre e informado de la persona interesada, basado en la información adecuada. Cuando proceda, el consentimiento debería ser expreso y la persona interesada podrá revocarlo en todo momento y por cualquier motivo, sin que esto entrañe para ella desventaja o perjuicio alguno". 18 DREWs, Jürgen. In Quest of Tomorrow's Medicines. Nueva York: Sprinter, 1998.

BIOÉTICA,

MEDICAMENTOS,

CONFLICTO DE

INTERESES Y

CONTROL DE

CALIDAD

BIOETHICS,

MEDICINES,

INTERESTS

CONFLICT AND

QUALITY CONTROL 
la revista Prescrire señala que el costo más frecuentemente citado es de 802 millones de dólares por medicamento y que aun dicho costo resulta exagerado porque «ha sido estimado por un instituto, en buena parte financiado por las firmas farmacéuticas, a partir de datos confidenciales proporcionados por dichas firmas». La revista agrega que los costos representan en realidad la mitad del monto estimado ${ }^{19}$.

En el caso de los medicamentos huérfanos ${ }^{20}$, los costos de investigación son mucho más bajos. iA qué se debe esta diferencia? Como explicamos anteriormente, una de las razones reside en el hecho de que parte del financiamiento de la promoción del medicamento (la llamada fase 4 de los ensayos clínicos) es contabilizada en la rúbrica de «costos de investigación y desarrollo». Muy a menudo, la llamada fase 4 tiene como objetivo principal incitar y habituar al médico que evalúa el nuevo medicamento a prescribirlo. Los medicamentos huérfanos no necesitan campañas de promoción sumamente costosas porque sus posibles «clientes» son limitados. Por el contrario, los nuevos medicamentos necesitan campañas de promoción muy onerosas para poder imponerse en el mercado, más aun si esos nuevos medicamentos poseen un modesto avance terapéutico.

\subsection{La practica terapéutica}

Desde el punto de vista ético, la práctica terapéutica debe guiarse por los principios fundamentales de beneficencia, de no maleficencia, de autonomía, de consentimiento y de justicia. La noción de beneficencia aplicada a los medicamentos supone que todo tratamiento debería potenciar al máximo los beneficios directos e indirectos para los pacientes, tratando de reducir lo más posible el riesgo de efectos nocivos ${ }^{21}$. Sin embargo, los medicamentos pueden producir efectos adversos.

El principio de autonomía defiende el deber de respetar la autodeterminación del paciente. Este concepto es dependiente del consentimiento informado, ya que el paciente no podrá tomar una decisión justa y eficaz si no ha recibido la información adecuada y necesaria. En un gran número de países, esta práctica no está desarrollada y los pacientes no son informados por los profesionales de salud sobre los efectos nocivos de los medicamentos. Además, estos efectos son sistemáticamente ocultados por los visitadores médicos, y en algunos casos, las propias firmas farmacéuticas disimulan durante un cierto tiempo los efectos nocivos graves de ciertos medicamentos.

19 «Coût de recherche et développement du médicament: la grande illusion». Prescrire, XXIII, 244 (2003), pp. 782-787.

20 Los medicamentos huérfanos son aquellos utilizados para el tratamiento de enfermedades cuyo número de pacientes es menor de 200000.

21 Artículo 4 de la Declaración Universal sobre Bioética y Derechos Humanos, adoptada por la Conferencia General de la Unesco, octubre de 2005. 
Este período de tiempo puede ser trágico para un cierto número de pacientes como fue el caso del benfluorex, que fue retirado del mercado en 2009, seis años después de una publicación científica española que señalaba la asociación entre el benfluorex y la aparición de lesiones valvulares cardiacas ${ }^{22}$.

\section{LOS CONFLICTOS DE INTERESES}

El conflicto de intereses ${ }^{23}$ se define como un conjunto de condiciones y circunstancias que pueden influenciar indebidamente el juicio profesional con relación al interés primario (bienestar y tratamiento del paciente, validez de la investigación) por un interés secundario (provecho económico, afán de notoriedad, prestigio, reconocimiento y promoción profesional). El conflicto de intereses se refiere sobre todo a una «situación» y no necesariamente a un «comportamiento». Por esta razón, difiere esencialmente de la «falta científica» (plagio, invención o distorsión de resultados, publicaciones redundantes, etcétera), la cual afecta la seriedad y calidad científica.

El principal interés de un profesional de la salud es actuar por el bien de las personas y la comunidad a la que sirve. El interés secundario frecuentemente no tiene ninguna relación con la misión fundamental del profesional. Este es generalmente el producto de un enfrentamiento entre diversas presiones, ya que dicho profesional puede estar confrontado a un conflicto de intereses de naturaleza política, académica, religiosa o personal. Sin embargo, muy a menudo el interés secundario es de naturaleza financiera ${ }^{24}$.

Existe pues, conflicto de intereses cuando una persona tiene la obligación moral de actuar en nombre de otros y esta acción se ve comprometida por los estrechos vínculos que tiene con un tercero.

En los países donde no existen reglamentos sobre el tema, los conflictos de intereses en materia biomédica pueden ser de diversas formas ${ }^{25}$ :

- regalos directos e indirectos;

- patrocinio de actividades culturales y científicas;

- formación continuada (seminarios, mesas redondas, conferencias) financiada por la industria farmacéutica;

- posesión de acciones o títulos financieros;

22 RAFEL RiBERA, Josefa \& otros. "Valvulopatía cardíaca asociada al uso de benfluorex». Revista Española de Cardiología, LVI, 2 (2003), pp. 215-216.

23 THOMPSON, Dennis. «Understanding Financial Conflicts of Interest». The New England Journal of Medicine, CCCXXIX, 8 (1993), pp. 573-576.

24 LA ROSA, Emilio. La fabricación de nuevas patologías: de la salud a la enfermedad. Lima: FCE, 2009.

25 MoYNIHAN, Ray. «Who Pays for the Pizza? Redefining the Relationships between Doctors and Drug Companies. 1: Entanglement». BMJ, CCCXXVI, 7400 (2003), pp. 1189-1192. 
- investigación financiada por la industria farmacéutica;

- financiamiento de cátedras académicas o de asociaciones de profesionales;

- actividades de consultor;

- redacción por parte de terceros de artículos «científicos».

La costumbre de los regalos no solo está muy extendida en muchos países, sino que también es considerada como «normal», debido a que se encuentra muy anclada en la vida profesional, sin permitir ningún tipo de cuestionamiento. Así pues, un porcentaje significativo de médicos que reciben regalos de las empresas farmacéuticas niega su influencia, a pesar de las evidencias que demuestran lo contrario ${ }^{26}$.

Las cenas y los viajes para asistir a conferencias, congresos, simposios y coloquios son prácticas comunes en muchos países. Sin embargo, se sabe que dichas prácticas influencian y aumentan la prescripción de medicamentos pertenecientes al laboratorio que financia esas actividades ${ }^{27}$. Este tipo de práctica también es considerado normal, a tal punto que la mayoría de médicos desean y esperan que las empresas farmacéuticas patrocinen sus reuniones de formación permanente ${ }^{28}$.

De la misma manera, muchas sociedades médico-científicas dependen, para la publicación de sus revistas y suplementos, del patrocinio y de la ayuda financiera de la industria ${ }^{29}$. Esta ayuda se da generalmente a través de la publicidad farmacéutica que dichas revistas insertan en sus páginas ${ }^{30}$. Este tipo de dependencia tiene generalmente algunas consecuencias, tal como lo precisa Lisa Bero, se ha demostrado que los suplementos de las revistas que están patrocinadas por la industria farmacéutica tienden a hacer más promoción a sus patrocinadores en comparación con otros suplementos que no se benefician de este tipo de ayuda ${ }^{31}$.

Dar o recibir regalos tiene un significado preciso y un carácter muy importante en muchas culturas, incluso un regalo insignificante exige lealtad y amistad ${ }^{32}$. Ciertos estudios sobre la toma de decisiones

26 WAZANA, Ashley. Ob. cit. Ha sido demostrada una correlación y una interacción entre las acciones de comunicación y marketing farmacéutico y las preferencias de los médicos con relación a los nuevos medicamentos (que no han demostrado una ventaja o beneficio adicional con respecto a los medicamentos existentes), que se traduce en una disminución de la prescripción de genéricos y en el aumento de los gastos en medicamentos.

27 LEXCHIN, Joel. «Interactions between Physicians and the Pharmaceutical Industry: What Does the Literature Say?» Canadian Medical Association Journal, CXLIX, 10 (1993), pp. 1401-1407.

28 lbídem.

29 Ver MOYNIHAN, Ray. Ob. cit.

30 Smith, R. «Medical Journals and Pharmaceutical Companies: Uneasy Bedfellows». BMJ, CCCXXVI (2003), pp. 1202-1205.

31 Bero, Lisa \& otros. «The Publication of Sponsored Symposiums in Medical Journals». The New England Journal of Medicine. CCCXXVII (1992), pp. 1135-1140.

32 KRIMSKY, Sheldon. «Small Gifts, Conflicts of Interest, and the Zero-Tolerance Threshold in Medicine». The American Journal of Bioethics, III, 3 (2003), pp. 50-52. KATZ, Dana \& otros. "All Gifts Large and 
muestran que el interés personal afecta el proceso de reflexión. Cuando los investigadores solicitan a las personas que expliquen la falta de objetividad, estas afirman que ninguna recompensa los hará perder su objetividad. Por el contrario, sus adversarios serían mucho más influenciables. En realidad, da la impresión que las personas encuestadas han subestimado considerablemente su pérdida de objetividad.

Dichos trabajos muestran también que esos mecanismos son inconscientes e indirectos. El interés personal modifica la forma en que los individuos buscan y analizan la información ${ }^{33}$ y los médicos e investigadores no son inmunes a este tipo de reacción. Así, por ejemplo, los internos y residentes de un hospital que participaron en eventos científicos organizados o patrocinados por un laboratorio farmacéutico tuvieron una mayor predisposición para prescribir medicamentos comercializados por el patrocinador, en comparación con aquellos que no asistieron a dichos eventos. Un estudio muestra que de los veinte médicos que asistieron a seminarios organizados por dos empresas farmacéuticas, diecinueve afirmaron antes de los seminarios que esas actividades no modificarían sus métodos de trabajo. Sin embargo, se constató, después de los seminarios, que dichos médicos prescribían con mayor frecuencia los medicamentos fabricados por esas empresas.

Los investigadores concluyen que cualquier medida para controlar la influencia de las compañías farmacéuticas (limitar los regalos, sensibilizar a los médicos u obligarlos a declarar cualquier regalo recibido) está condenada al fracaso porque se basa en un modelo falso del comportamiento humano. Su conclusión es simple: hay que prohibir todo tipo de regalos que la industria farmacéutica ofrece a los médicos ${ }^{34}$. En algunos países estos regalos son considerados como casos de corrupción ${ }^{35}$.

Un documento de la Organización Mundial de la Salud subraya que: «Las empresas se enfrentan a una enorme presión para ganar y retener una parte del mercado» ${ }^{36}$ creando un conflicto de intereses inmanente entre los fines comerciales legítimos de esas empresas y las necesidades médicas, económicas y sociales de los proveedores de salud y del público.

El concepto de conflicto de intereses no es sinónimo de juicio sobre la honestidad de la persona, sino que expresa más bien una injerencia latente. Por cierto, a menudo es difícil o imposible detectar una influencia

Small: Toward an Understanding of Pharmaceutical Industry Gift-Giving». The American Journal of Bioethics. III, 3 (2003), pp. 39-46.

33 DANA, Jason \& George LOWESTEIN, «A Social Science Perspective on Gifts to Physicians from Industry". The Journal of the American Medical Association, CCXC, 2 (2003), pp. 252-255. 173). 
nefasta ${ }^{37}$. La magnitud actual de las interacciones entre el médico y la industria farmacéutica afectan la prescripción y el comportamiento profesional ${ }^{38}$; esta situación debe ser tratada con seriedad por los gobiernos, los profesionales y las sociedades científicas.

\section{EL CONTROL DE CALIDAD EN MEDICINA}

El principio de no maleficencia exige que los profesionales y las autoridades de salud deben crear las condiciones adecuadas para evitar errores que puedan perjudicar al paciente no solamente en materia de tratamiento, sino también de los procedimientos diagnósticos. Dentro de estos procedimientos se encuentran los exámenes de laboratorio y radiológicos. En este sentido, es necesario señalar que en el Perú no existe aún un control de calidad sistemático, periódico, obligatorio e independiente de los laboratorios de exámenes biológicos ni de la radiología. En el caso de los laboratorios, la ausencia de control de calidad que cumpla los requisitos ya mencionados puede generar un aumento del número de resultados falsos. Es decir, un paciente puede ser diagnosticado de una anomalía inexistente (falso positivo) o no ser diagnosticado de un problema existente (falso negativo). En el primer caso, el paciente recibirá un tratamiento por una enfermedad inexistente y en el segundo caso la persona será declarada sana cuando en realidad presenta una anomalía. Ambas posibilidades son perjudiciales para el paciente y son contrarias al principio fundamental de no maleficencia.

Lo mismo podemos decir en relación con la falta de control de calidad de la radiología y de los aparatos de radioterapia. Esta carencia puede ser grave cuando se trata del diagnóstico y/o tratamiento de ciertas enfermedades malignas, como puede ser el del cáncer de mama: un mamógrafo que no es sometido a un control sistemático y periódico corre el riesgo de no detectar tumores de pequeña talla (diámetro inferior a diez milímetros), perdiendo una oportunidad crucial de diagnóstico y tratamiento exitoso, en la medida en que generalmente los tumores pequeños no dan metástasis, es decir, están localizados. Este tipo de tumores tiene un porcentaje muy elevado de curación luego del tratamiento quirúrgico.

\section{CONCLUSIÓN}

Es importante que la publicidad farmacéutica sea contrabalanceada por un programa público de información en materia de medicamentos que sea de fácil acceso para los médicos. 
En pos del beneficio terapéutico, de manera semejante, los médicos deberían privilegiar los medicamentos existentes y no los nuevos, debido a dos elementos esenciales: el farmacológico, el medicamento existente posee una mayor y mejor fármaco-vigilancia que el nuevo; el económico, el fármaco existente tiene un precio considerablemente menor que el nuevo.

Hay conflicto de interés cuando la industria farmacéutica financia congresos, conferencias, viajes, regalos, etcétera, a los médicos y estos conflictos son contrarios a los principios elementales de ética médica.

Las autoridades sanitarias deberían crear las condiciones necesarias para poner en práctica un control obligatorio, sistemático y periódico de los laboratorios clínicos y de los centros de radiología y radioterapia.

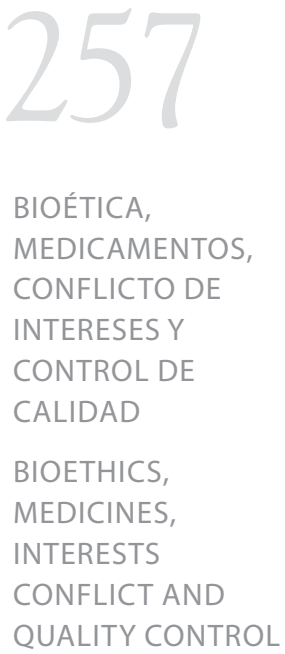

